\title{
ELEMENTS OF MINIMAL BREADTH IN FINITE $p$-GROUPS AND LIE ALGEBRAS
}

\author{
AVINOAM MANN
}

(Received 4 February 2005; revised 15 May 2005)

Communicated by E. A. O'Brien

\begin{abstract}
Let $G$ be a finite $p$-group, and let $M(G)$ be the subgroup generated by the non-central conjugacy classes of $G$ of minimal size. We prove that this subgroup has class at most 3. A similar result is noted for nilpotent Lie algebras.
\end{abstract}

2000 Mathematics subject classification: primary 20D15, 20E45, $17 \mathrm{~B} 30$.

This note is concerned with the influence of the conjugacy class sizes of a finite group on its structure and the analogous problem for Lie algebras. The only groups that we consider are finite $p$-groups, and the letter $G$ is reserved to denote such a group. Let $G$ be a finite $p$-group, and let $1=n_{1}<n_{2}<\cdots<n_{k}$ be the sizes of its conjugacy classes. We refer to classes of size $n_{2}$ as minimal classes, and to their elements as minimal elements. We also write $n_{i}=p^{b_{i}}$, and if $x$ lies in a class of size $n_{i}$, we say that $x$ has breadth $b_{i}$, and write $b_{G}(x)=b_{i}$ (the index $G$ will often be omitted). In [7], Ishikawa has shown that if $k=2$, then $\operatorname{cl}(G) \leq 3$. A simplification of his proof, by Isaacs, appears in [1]. In [8] we pointed out that a slight variation on Ishikawa's argument proves that if $G$ is generated by minimal elements, then $\operatorname{cl}(G) \leq 3$, and conjectured that in any $p$-group the subgroup, $M(G)$ say, generated by the minimal elements has its class bounded by a function of $p$ only. We were able to prove this for $p=2$, with the bound 2, and derived from it that 2-groups with $k=3$ are metabelian. Here we prove a strong form of the full conjecture, showing that $\operatorname{cl}(M(G)) \leq 3$ always. This provides, in particular, a new proof of Ishikawa's theorem, and we also deduce from it that 3-groups with $k=3$ have derived length at most 4.

(C) 2006 Australian Mathematical Society 1446-7887/06 $\$ A 2.00+0.00$ 
One of the difficulties in Ishikawa's theorem is that the assumption that all noncentral classes have the same size is inherited neither by subgroups nor by factor groups, and so induction is not applicable. As a simple example of this problem, consider the group $G$ with presentation $\left\langle x, y, z \mid x^{p}=y^{p}=z^{p}=1\right\rangle$, and all commutators of weight 3 being the identity. This is a group of class 2 and order $p^{6}$, in which the non-central elements have $p^{2}$ conjugates each. However, in $H=G /\langle[x, y]\rangle$ there are classes of both sizes $p$ and $p^{2}$. More generally, let $G$ be generated by $x_{1}, \ldots, x_{n}$, with all generators of order $p$, and all commutators of weight 3 trivial. Then

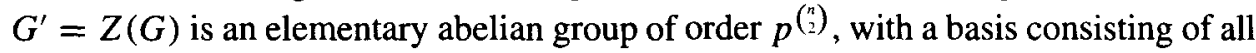
commutators $\left[x_{i}, x_{j}\right], i<j$. It is easy to see that if $x \notin Z(G)$, then $C(x)=\langle x, Z(G)\rangle$, and thus all non-central elements of $G$ have breadth $n-1$. In [4] there are constructed $p$-groups, of class 2 and generated by elements of order $p$, with an arbitrary set (containing 1) of $p$-powers as the set of class sizes. Such a group is a factor group of the above $G$, if $n$ is large enough. Thus there can be no restriction on the number of class sizes of factor groups of groups with just two sizes. We avoid that problem by focusing on the breadth of a single element.

Similar to the group case, let $L$ be a finite-dimensional nilpotent Lie algebra. We then write $0=b_{1}<b_{2}<\cdots<b_{k}$ for the codimensions of the centralizers (annihilators) of the elements of $L$. An element whose centralizer has codimension $b_{2}$ is termed minimal. Then one can prove, by arguments which are almost identical to the group case, results analogous to Theorems 1, 5, and 7, below. Indeed, some of the proofs are even slightly shorter, because we do not need to consider $p$ th powers. We do not spell out the proofs, just state the analogue of Theorem 5.

THEOREM L. Let $L$ be a finite-dimensional nilpotent Lie algebra, and write $M(L)$ for the subalgebra generated by the non-central elements with a centralizer of maximal dimension. Then $\operatorname{cl}(M(L)) \leq 3$.

We recall that Lie algebras analogues of Ishikawa's theorem, and other related results, were proved earlier in [1] (of course Theorem L provides another proof of Ishikawa's theorem for Lie algebras). It may be thought that, analogously to the fact that in 2-groups $M(G)$ has class at most 2, in Lie algebras of characteristic 2 the subalgebra $M(L)$ is of class at most 2 . However, that does not hold. Consider, over any field, a 5-dimensional Lie algebra $L$, with basis $x, y, z, u, v$, relations $[x, y]=$ $-[y, x]=z,[x, z]=-[z, x]=u,[y, z]=-[z, y]=v$, and where all the other commutators are 0 . Then $L$ is an algebra of class 3 , in which all non-central elements have a centralizer of dimension 3 . The group analogues of this example, groups of order $p^{5}$ and class 3 , with $\left|G^{\prime}\right|=|G: Z(G)|=p^{3}$, exist only for odd $p$ (see [6] for many examples, and partial classification, of $p$-groups with $k=2)$.

We use $C_{G}(X)$ and $N_{G}(X)$, or $C(X)$ and $N(X)$ when there is no danger of confusion, for the centralizer and normalizer of the element, or subgroup, $X$, in $G$. We denote by 
$Z_{i}(G)$ and $\gamma_{i}(G)$ the upper and lower central series, $Z(G), G^{\prime}$, and $\Phi(G)$ the centre, derived subgroup, and Frattini subgroup, respectively, and $\mathrm{cl}(G)$ the nilpotence class of $G$. Also, if $N \triangleleft G$, then $Z(G \bmod N)$ is defined by $Z(G \bmod N) / N=Z(G / N)$.

Our main technical result is as follows.

THEOREM 1. Let $K$ be a normal abelian subgroup of $G, x$ be a non-central element of $G$ of breadth $b$, and $y \in[K, x]$. Then $b(y)<b$.

LeMma 2. Let $x \in H \leq G, N \triangleleft G$, and $|G: H|=|N|=p$. Then $b(x)-1 \leq$ $b_{G / N}(x N) \leq b(x)$ and $b(x)-1 \leq b_{H}(x) \leq b(x)$. If $C(x) \leq H$, then $b_{H}(x)=$ $b(x)-1$.

PROOF. The first inequality follows from the facts that the conjugacy class of $x$ maps onto the conjugacy class of $x N$, and that at most $p$ elements of $G$ map to the same element of $G / N$. The other claims are obvious.

LEMMA 3. Using the notation above, assume that $n_{2}>p$, and let $N \triangleleft G$ have order $p$. Then $Z(G \bmod N)=Z(G)$.

Proof. Let $x \in Z(G \bmod N)$. Then all conjugates of $x$ lie in $x N$, so $x$ has at most $p$ conjugates. The assumption $n_{2}>p$ shows that $x$ is central.

PROOF OF THEOREM 1. Naturally we may assume that $K \nless C(x)$. If $b(x)=1$, then $|G: C(x)|=p$, therefore $G=K C(x)$, and $C(x) \triangleleft G$, and so also $Z(C(x)) \triangleleft G$. Since $x \in Z(C(x))$, we have $[K, x] \leq K \cap Z(C(x)) \leq Z(G)$, as desired. Now assume that $b(x)>1$, and apply induction on $b=b(x)$. We can find a subgroup $C(x)<$ $H \leq K C(x)$ such that $|H: C(x)|=p$. Then $C(x) \triangleleft H$ and $H=C(x)(H \cap K)$, and we can find an element $z \in K$ such that $z \in N(C(x))-C(x)$ and $z^{p} \in C(x)$. Then $z$ normalizes $Z(C(x))$, which contains $x$, and therefore $1 \neq[x, z] \in Z(C(x)) \cap K$. Thus $C([x, z]) \geq K C(x)$, so $C([x, z])$ contains $C(x)$ properly, and $b([x, z])<b$. We now have two possibilities.

(I) $[x, z] \in Z(G)$. In that case $\operatorname{cl}(\langle x, z\rangle) \leq 2$, and so $[x, z]^{p}=\left[x, z^{p}\right]=1$, and $[x, z]$ has order $p$. Write $N=\langle[x, z]\rangle$. In $G / N$ the coset $x N$ commutes with both $C(x) / N$ and $z N$, and so $b_{G / N}(x N)<b_{G}(x)$. It follows from Lemma 2 that $x N$ has breadth $b(x)-1$. By induction $b_{G / N}(y N)<b-1$, and by Lemma $2, b(y)<b$.

(II) $[x, z] \notin Z(G)$. Then $K C(x) \neq G$. Let $L$ be a maximal subgroup containing $K C(x)$. Then $b_{L}(x)=b-1$, and by induction $b_{L}(y)<b-1$, implying $b_{G}(y)<b$.

An obvious but important corollary is as follows. 
COROLLARY 4. Let $K$ be a normal abelian subgroup of $G$. Then $[K, M(G)] \leq$ $Z(G)$.

THEOREM 5. $\operatorname{cl}(M(G)) \leq 3$ and $\operatorname{cl}(M(G) / Z(G)) \leq 2$. If $p=2$, then $\mathrm{cl}(M(G)) \leq$ 2 and $M(G) / Z(G)$ is abelian.

PROOF. Write $M=M(G)$. Let $K$ be maximal among the normal abelian subgroups of $G$ that are contained in $M$. Then $C_{M}(K)=K$. By Corollary $4,[M, K] \leq Z(G) \leq$ $Z(M)$, and so $K \leq Z_{2}(M)$. However, then $M^{\prime} \leq C_{M}(K)=K$, that is, $M^{\prime} \leq Z_{2}(M)$ and $\operatorname{cl}(M) \leq 3$. Also $\left[M^{\prime}, M\right] \leq[K, M] \leq Z(G)$, and so $\operatorname{cl}(M / Z(G)) \leq 2$.

That $\operatorname{cl}(M(G)) \leq 2$ if $p=2$ is Theorem 4 of [8]. That $M(G) / Z(G)$ is abelian in that case will be proved following the statement of Theorem 6 below. We first recall a definition and a result from [8].

DEFINITION. Let $G$ be a $p$-group. The centralizer equality subgroup $D(G)$ of $G$ is the subgroup generated by all elements of $G$ satisfying $C(x)=C\left(x^{p}\right)$.

Obviously $D(G) \geq Z(G)$, and easy examples show that both equality and inequality of these two subgroups occur. The applicability of this subgroup in our context follows from the obvious fact that if $x$ is a minimal element, then either $x^{p} \in Z(G)$, or $x$ is one of the generating elements of $D(G)$. If $D(G) \leq H \leq G$, then $D(G) \leq D(H)$. The next result, which is [8, Theorem 7], shows that $D(G)$ always has a large centralizer. For its statement, recall that a left $n$-Engel element is an element $x$ such that $[y, x, \ldots, x]=1$ for all $y \in G$, where $x$ occurs in the commutator $n$ times.

THEOREM 6. Let $G$ be a p-group. then the following statements are true.

(a) $D(G)$ is abelian.

(b) If $D(G) \leq H \leq G$, and $\mathrm{cl}(H) \leq p$, then $D(G) \leq Z(H)$.

(c) $C\left(D(G)\right.$ ) contains $Z_{p}(G)$, as well as all normal subgroups of $G$ of class less than $p$, all elements of breadth less than $p$, and all left $p$-Engel elements.

(d) Let $c<p$. If $N$ is maximal among the normal subgroups of $G$ of class $c$, then $D(G) \leq N$.

(e) $D(G) \leq Z J(G)$.

Here we recall that $J(G)$ is the Thompson subgroup of $G$, the subgroup generated by all abelian subgroups of maximal order, and $Z J(G)$ is its centre, which is equal to the intersection of all these abelian subgroups. Recall also that various subgroups with properties similar to those of $J(G)$ have been defined; we have in mind the two $K$-subgroups of Glauberman [3, Section X.8] and the Puig subgroup $L(G)$ [2, Appendix B]. It is not difficult to see that $D(G)$ lies in the centre of all three of these subgroups. 
Now return to Theorem 5. It was proved in [8, Proposition 6] that the minimal elements of a 2-group $G$ lie either in $D(G)$ or in $Z_{2}(G)$. Therefore $M(G) / Z(G)$ is abelian, being a subgroup of the abelian group $D(G) Z_{2}(G) / Z(G)$.

The following result may be considered as a 'local' version of Theorem 5 .

THEOREM 7. Let $x$ be a minimal element. Then $c l\langle x\rangle^{G} \leq 2$. If $p=2$, then $\langle x\rangle^{G}$ is abelian.

PROOF. We will prove a stronger statement, that $\langle x\rangle^{G} Z(G) / Z(G)$ is abelian. First, let $b(x)=1$. Then $|G: C(x)|=p$, so $C(x) \triangleleft G$ and $Z(C(x)) \triangleleft G$. Since $x \in Z(C(x))$, $\langle x\rangle^{G}$ is abelian. Next, we assume that $b(x)>1$, and induct on $b(x)$. Let $K$ be a maximal normal abelian subgroup of $G$. Then $C(K)=K$. If $x \in K$, then $\langle x\rangle^{G} \leq K$, so $\langle x\rangle^{G}$ is abelian. If $x \notin K$, then $K \notin C(x)$, and as in the proof of Theorem 1 , we can find an element $y \in K$, such that $[y, x]$ is a central element of order $p$, and if $N=\langle[x, y]\rangle$, then in $G / N$ the element $x N$ is minimal, of breadth $b(x)-1$. By induction $\langle x\rangle^{G}$ is abelian, modulo $Z(G \bmod N)$. However, $Z(G \bmod N)=Z(G)$, by Lemma 3.

If $p=2$, then [8, Proposition 6] shows that either $x \in D(G)$ or $x \in Z_{2}(G)$. Thus $\langle x\rangle^{G}$ is contained either in the abelian group $D(G)$ or in the abelian group $\langle x, Z(G)\rangle$.

Combining the last theorem with Theorem 6 (c) yields the following addition to Theorem 6 .

COROLLARY 8. $D(G)$ centralizes all minimal elements.

In [8] we pointed out that if $G$ has $k$ class sizes, and $x \in G$, then $x^{p^{k-1}} \in D(G)$. In particular, $G$ is an extension of an abelian group by one of exponent $\leq p^{k-1}$ (if $p=2$, the exponent can be taken to be $2^{k-2}$ ). Analogously we have the following result.

COROLLARY 9. If $G$ has $k$ class sizes, then $G$ is an extension of a group of class 3 by a group of exponent $p^{k-2}$.

ProOF. Let $x \in G$, and consider $x, x^{p}, \ldots, x^{p^{k-1}}$. If two of these elements have the same centralizer, these two elements lie in $D(G)$, and in particular $x^{p^{k-2}} \in D(G)$. If no two of these powers have the same centralizer, they represent all the class sizes, so $x^{p^{k-1}}$ is central, and $x^{p^{k-2}}$ is minimal. In either case $x^{p^{k-2}} \in D(G) M(G)$, and the last subgroup is of class 3 , by Theorem 5 and Corollary 8 .

Since groups of exponent 3 are of class 3 and metabelian [5, Chapter 18], we obtain the following. 
COROLLARY 10. A 3-group with just three class sizes is an extension of a group of class 3 by a group of class 3 , and has derived length of at most 4 .

\section{Acknowledgements}

I am indebted to Y. Berkovich for several helpful suggestions.

\section{References}

[1] Y. Barnea and I. M. Isaacs, 'Lie algebras with few centralizer dimensions', J. Algebra 259 (2003), 284-299.

[2] H. Bender and G. Glauberman, Local analysis for the odd order theorem (Cambridge University Press, Cambridge, 1994).

[3] N. Blackburn and B. Huppert, Finite groups III (Springer, Berlin, 1982).

[4] J. Cossey and T. O. Hawkes, 'Sets of p-powers as conjugacy class sizes', Proc. Amer. Math. Soc. $128(2000), 49-51$.

[5] M. Hall, The theory of groups (Macmillan, New York, 1959).

[6] K. Ishikawa, 'Finite $p$-groups up to isoclinism, which have only two conjugacy lengths', J. Algebra 220 (1999), 333-345.

[7] —_ 'On finite $p$-groups which have only two conjugacy lengths', Israel J. Math. 129 (2002), 119-123.

[8] A. Mann, 'Groups with few class sizes and the centraliser equality subgroup', Israel J. Math. 142 (2004), 367-380.

Institute of Mathematics

Hebrew University

Jerusalem 91904

Israel

e-mail: mann@math.huji.ac.il 